\title{
Copulas with fractal supports
}

\author{
Gregory A. Fredricks ${ }^{\mathrm{a}, *}$, Roger B. Nelsen ${ }^{\mathrm{a}}$, José Antonio Rodríguez-Lallena ${ }^{\mathrm{b}}$ \\ ${ }^{a}$ Department of Mathematical Sciences, Lewis \& Clark College, Portland, OR 97219, USA \\ ${ }^{\mathrm{b}}$ Departamento de Estadística y Matemática Aplicada, Universidad de Almería, 04120 La Cañada de San Urbano, Almería, Spain
}

Received July 2004 ; received in revised form August 2004; accepted 15 December 2004

\begin{abstract}
Using an iterated function system, the authors construct families of copulas whose supports are fractals. In particular, they show that the members of one family have supports with arbitrary Hausdorff dimensions in the interval $(1,2)$. They also employ those copulas to construct more general bivariate distribution functions with fractal supports.

(C) 2005 Elsevier B.V. All rights reserved.

Keywords: Copula; Fractal; Iterated function system
\end{abstract}

\section{Introduction}

In this paper we establish the existence of copulas whose supports are fractals and prove

Theorem 1. For each $s \in(1,2)$, there exists a copula whose support is a fractal with Hausdorff dimensions.

Section 2 contains background information. We introduce the construction technique in Section 3 and prove Theorem 1 in Section 4. We obtain a generalization of Theorem 1 to distribution functions with more general margins in Section 5.

\footnotetext{
* Corresponding author.

E-mail address: fredrix@1clark.edu (G.A. Fredricks).
}

\section{Preliminaries}

A copula is a bivariate distribution function whose margins are uniform on $I=[0,1]$. For an introduction to copulas see Nelsen (1999). The Borel measure on $I^{2}$ corresponding to a copula $C$ is denoted by $\mu_{C}$. We use Mandelbrot's original definition of a fractal (see Edgar, 1990 ,p. 179); specifically, a fractal is a set whose topological dimension is less than its Hausdorff dimension. For an introduction to fractals, including Hausdorff and topological dimensions, see Edgar (1990).

Most of our results follow from general techniques relating to iterated function systems (IFSs henceforth), for which Edgar $(1990,1998)$ offer excellent introductions. We have chosen these and Barnsley (1989) as our references. A hyperbolic IFS consists of a complete metric space $(X, d)$ together with a finite set of contraction mappings $\left(w_{n}: X \rightarrow X\right)$. The hyperbolic IFS $\left\{X ;\left(w_{n}\right)\right\}$ satisfies Moran's open set 
condition if there exists a nonempty open subset $U$ of $X$ for which $w_{n}(U) \cap w_{m}(U)=\emptyset$ whenever $n \neq m$ and $w_{n}(U) \subseteq U$ for all $n$.

Proposition 1 (Barnsley, 1989, p. 131 and Edgar, 1990, pp. 107 and 161). If $\left\{X ;\left(w_{n}\right)\right\}$ is a hyperbolic $I F S$, then there exists a unique nonempty compact subset $K$ of $X$ for which $K=\bigcup_{n} w_{n}(K)$. Moreover, if each $w_{n}$ is a similarity and Moran's open set condition is satisfied, then the Hausdorff dimension $s$ of $K$ is given implicitly by the equation $\sum_{n} c_{n}^{s}=1$, where $c_{n}$ is the similarity ratio of $w_{n}$.

$K$ is called the invariant set of $\left\{X ;\left(w_{n}\right)\right\}$. A hyperbolic IFS with probabilities is a hyperbolic IFS $\left\{X ;\left(w_{1}, \ldots, w_{N}\right)\right\}$ together with an ordered set of positive numbers $\left(p_{1}, \ldots, p_{N}\right)$ for which $\sum_{i=1}^{N} p_{i}=1$. Each hyperbolic IFS with probabilities $\left\{X ;\left(w_{n}\right),\left(p_{n}\right)\right\}$, with $X$ compact, defines a Markov operator $M$ : $\mathcal{P}(X) \rightarrow \mathcal{P}(X)$, where $\mathcal{P}(X)$ denotes the set of normalized Borel measures on $X$, by

$M(v)=\sum_{n} p_{n}\left(\nu \circ w_{n}^{-1}\right)$.

Proposition 2 (Barnsley, 1989, p. 139). If $\left\{X ;\left(w_{n}\right),\left(p_{n}\right)\right\}$ is a hyperbolic IFS with probabilities and $X$ is compact, then there exists a unique $\mu \in \mathcal{P}(X)$ for which $M(\mu)=\mu$.Moreover, the support of $\mu$ is the invariant set of $\left\{X ;\left(w_{n}\right)\right\}$.

$\mu$ is called the invariant measure of $\left\{X ;\left(w_{n}\right),\left(p_{n}\right)\right\}$.

\section{Transformation matrices and invariant copulas}

Definition 1. A transformation matrix is a matrix $T$ with nonnegative entries, for which the sum of the entries is 1 and no row or column has every entry zero.

We double subscript the entries of $T$ with the column index first and the rows ordered from bottom to top, e.g., $T=\left[\begin{array}{ll}t_{12} & t_{22} \\ t_{11} & t_{21}\end{array}\right]$,

to conform with the order of coordinates in $I^{2}$ (see Example 1). Each transformation matrix $T$ determines a subdivision of $I^{2}$ into subrectangles as follows. Let $p_{i}$ denote the sum of the entries in the first $i$ columns of $T$, let $q_{j}$ denote the sum of the entries in the first $j$ rows of $T$, and let $p$ and $q$ denote the partitions of $I$ that they form. The partitions $p$ on the horizontal axis and $q$ on the vertical axis determine an obvious partition of $I^{2}$ into subrectangles. We let $R_{i j}=\left[p_{i-1}, p_{i}\right] \times\left[q_{j-1}, q_{j}\right]$.

Example 1. For the transformation matrix

$T=\left[\begin{array}{ccc}0.1 & 0.2 & 0.3 \\ 0.4 & 0 & 0\end{array}\right]$,

we have $p=(0, .5, .7,1), q=(0, .4,1)$ and the subdivision of $I^{2}$ illustrated in Fig. 1 with $R_{i j}$ shaded whenever $t_{i j}>0$.

For a transformation matrix $T$ and a copula $C$, let $T(C)$ be the copula which, for each $(i, j)$, spreads mass $t_{i j}$ on $R_{i j}$ in the same (but rescaled) way in which $C$ spreads mass on $I^{2}$. Specifically, the copula $T(C)$ is given on $R_{i j}$ by

$$
\begin{aligned}
T(C)(u, v)= & \sum_{i^{\prime}<i, j^{\prime}<j} t_{i^{\prime} j^{\prime}}+\frac{u-p_{i-1}}{p_{i}-p_{i-1}} \sum_{j^{\prime}<j} t_{i j^{\prime}} \\
& +\frac{v-q_{j-1}}{q_{j}-q_{j-1}} \sum_{i^{\prime}<i} t_{i^{\prime} j} \\
& +t_{i j} C\left(\frac{u-p_{i-1}}{p_{i}-p_{i-1}}, \frac{v-q_{j-1}}{q_{j}-q_{j-1}}\right),
\end{aligned}
$$

where empty sums are defined to be zero.

A direct calculation establishes the following result on $T(C)$-volumes.

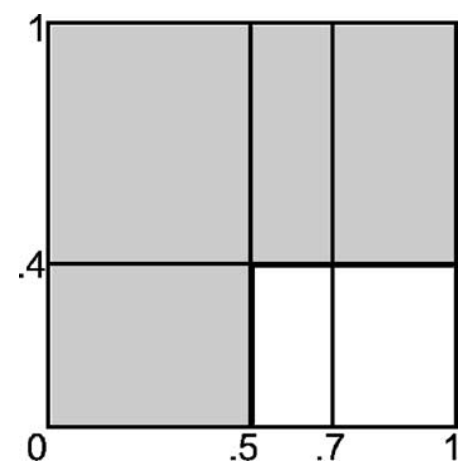

Fig. 1. Subdivision of $I^{2}$ given by the transformation matrix (1). 
Proposition 3. For each $(i, j)$ and each $(u, v) \in R_{i j}$, we have

$$
\begin{aligned}
& V_{T(C)}\left(\left[p_{i-1}, u\right] \times\left[q_{j-1}, v\right]\right) \\
& \quad=t_{i j} C\left(\frac{u-p_{i-1}}{p_{i}-p_{i-1}}, \frac{v-q_{j-1}}{q_{j}-q_{j-1}}\right) .
\end{aligned}
$$

Note that the support of $T(\Pi)$, where $\Pi(u, v)=u v$, is the union of all the rectangles $R_{i j}$ for which $t_{i j}>$ 0 , and that the support of $T(C)$ for any copula $C$ is contained in the support of $T(\Pi)$.

Example 2. For $T$ in Example 1 and $M(u, v)=$ $\min (u, v), T(M)$ is the copula which, for each $(i, j)$, spreads mass $t_{i j}$ uniformly on the diagonal of $R_{i j}$ (see Fig. 2).

If $T$ is a square matrix with exactly one nonzero entry in each row and each column, then $T(M)$ is a (straight) shuffle of $M$ (see Mikusiński et al., 1992, p. 64). Also, if $C$ is a copula, $n$ is a positive integer, and $T$ is the $n \times n$ matrix with coefficients given by

$$
\begin{aligned}
t_{i j}= & C\left(\frac{i}{n}, \frac{j}{n}\right)-C\left(\frac{i-1}{n}, \frac{j}{n}\right)-C\left(\frac{i}{n}, \frac{j-1}{n}\right) \\
& +C\left(\frac{i-1}{n}, \frac{j-1}{n}\right),
\end{aligned}
$$

then $T$ is a transformation matrix and $T(\Pi)$ is a checkerboard approximation to $C$ (see Li et al. (1997), p. 109).

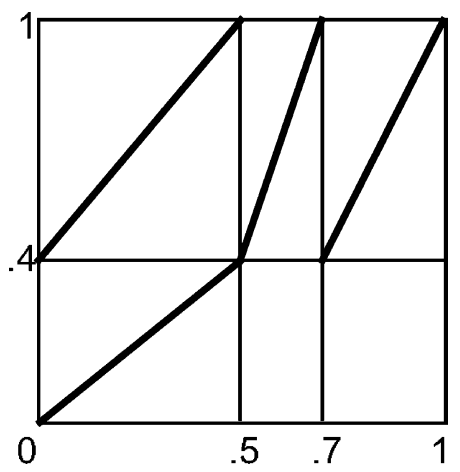

Fig. 2. Support of the copula $T(M)$, where $T$ is the transformation matrix given by (1).
Proposition 4. Let $T$ be a transformation matrix and let $C_{1}$ and $C_{2}$ be copulas. Then

(a) $T\left(C_{1}\right) \leq T\left(C_{2}\right)$ whenever $C_{1} \leq C_{2}$, where $\leq$ is defined pointwise, and

(b) $d\left\{T\left(C_{1}\right), T\left(C_{2}\right)\right\}=\max _{i, j}\left(t_{i j}\right) d\left(C_{1}, C_{2}\right)$, where $d$ denotes the sup metric.

Proof. Part (a) follows from the definition of $T(C)$. For (b), note that for each $(u, v) \in R_{i j}$,

$$
\begin{aligned}
& \left|T\left(C_{1}\right)(u, v)-T\left(C_{2}\right)(u, v)\right| \\
& =t_{i j} \mid C_{1}\left(\frac{u-p_{i-1}}{p_{i}-p_{i-1}}, \frac{v-q_{j-1}}{q_{j}-q_{j-1}}\right) \\
& \quad-C_{2}\left(\frac{u-p_{i-1}}{p_{i}-p_{i-1}}, \frac{v-q_{j-1}}{q_{j}-q_{j-1}}\right) \mid .
\end{aligned}
$$

Thus, $\quad \sup _{(u, v) \in R_{i j}}\left|T\left(C_{1}\right)(u, v)-T\left(C_{2}\right)(u, v)\right|=$ $t_{i j} d\left(C_{1}, C_{2}\right)$ and the result follows.

For a transformation matrix $T$ and a copula $C$, we now consider successive iterations of $T$. Define $T^{2}(C)=$ $T\{T(C)\}, T^{3}(C)=T\left\{T^{2}(C)\right\}$, etc. Note that each $T^{m}(C)$ is a copula and that $T^{m}(C)=\left(\otimes^{m} T\right)(C)$, where $\otimes^{m} T$ is the tensor product of $T$ with itself $m$ times.

Example 3. For the transformation matrix

$$
T=\left[\begin{array}{ccc}
0.1 & 0 & 0.1 \\
0 & 0.6 & 0 \\
0.1 & 0 & 0.1
\end{array}\right]
$$

(where $p=q=(0,0.2,0.8,1)$ ), the supports of $T(\Pi)$ and $T^{2}(\Pi)$ are pictured as shaded regions in Fig. 3 .
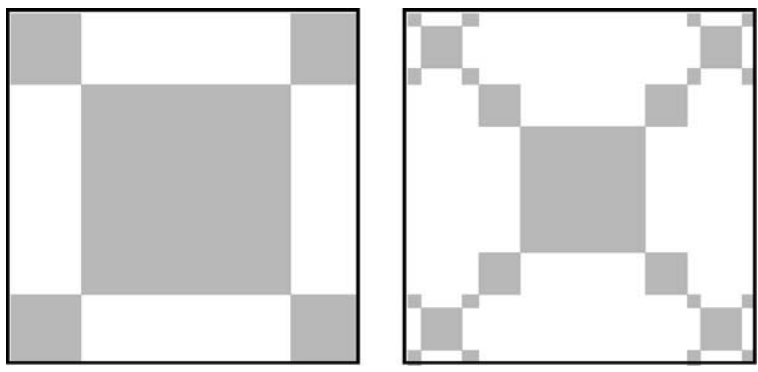

Fig. 3. Supports of the copulas $T(\Pi)$ and $T^{2}(\Pi)$, where $T$ is the transformation matrix given by (2). 
Note that if $T \neq[1]$ (the $1 \times 1$ matrix with entry 1$)$, then $\max _{i, j}\left(t_{i j}\right) \in(0,1)$ and hence, from (b) in Proposition 4 , that $T$ is a contraction mapping. Since the set of copulas endowed with the sup metric is a complete metric space, the Contraction-Mapping Theorem yield.

Theorem 2. For each transformation matrix $T \neq$ [1], there is a unique copula $C_{T}$ for which $T\left(C_{T}\right)=$ $C_{T}$. Moreover, $C_{T}$ is the limit of the sequence $\left\{C, T(C), T^{2}(C), \ldots, T^{m}(C), \ldots\right\}$ for any copula $C$.

Definition 2. A copula $C$ is invariant if $C=C_{T}$ for some transformation matrix $T$.

In the following example, we see that $\Pi, M$ and $W(u, v)=\max (u+v-1,0)$ are invariant.

Example 4. If $T=\left[\begin{array}{ll}0.25 & 0.25 \\ 0.25 & 0.25\end{array}\right]$, then $C_{T}=\Pi$. If $T=\left[\begin{array}{cc}0 & 0.5 \\ 0.5 & 0\end{array}\right]$, then $C_{T}=M$. If $T=\left[\begin{array}{cc}0.5 & 0 \\ 0 & 0.5\end{array}\right]$, then $C_{T}=W$. More generally, $C_{T}=\Pi$ if $T$ is a row or column matrix or if all the entries of $T$ are the same; $C_{T}=M$ if $T$ is square and all the nonzero entries of $T$ lie on the ascending diagonal; and $C_{T}=W$ if $T$ is square and all the nonzero entries of $T$ lie on the descending diagonal.

In addition, note that if $p=\left(p_{i}\right)$ and $q=\left(q_{j}\right)$ are any two partitions of $I$, then the matrix $T=\left(t_{i j}\right)$ with $t_{i j}=\left(p_{i}-p_{i-1}\right)\left(q_{j}-q_{j-1}\right)$ is a transformation matrix which gives rise to the partitions $p$ and $q$ and has $C_{T}=\Pi$.

\section{Self-similar copulas and the proof of Theorem 1}

Since the latter part of Theorem 2 tells us that the limit copula $C_{T}$ is independent of the "seed" copula used to generate the iterative sequence, we may study $C_{T}$ as the limit of the sequence $\left\{T^{m}(\Pi)\right\}$. For example, consider a transformation matrix $T$ which has at least one zero entry. If $T$ has $\ell$ nonzero entries, then the support $\sigma_{1}$ of $T(\Pi)$ consists of $\ell$ rectangles and the union of those rectangles has an area $A<1$. For each $m \geq 2$, the support $\sigma_{m}$ of $T^{m}(\Pi)$ consists of $\ell^{m}$ rectangles and has area $A^{m}$. Note that $\left(\sigma_{m}\right)$ is a nested sequence of subsets of $I^{2}$ and that the support of $C_{T}$ is contained in $\sigma=\bigcap_{m=1}^{\infty} \sigma_{m}$. Since $\sigma$ has Lebesgue measure zero, the support of $C_{T}$ also has Lebesgue measure zero, and thus, by definition, is a singular copula. We have proven

Theorem 3. If $T$ is a transformation matrix with at least one zero entry, then $C_{T}$ is singular.

For each transformation matrix $T=\left(t_{i j}\right)$, define $f_{i j}: I^{2} \rightarrow I^{2}$ by

$$
\begin{aligned}
& f_{i j}(u, v) \\
& \quad=\left\{p_{i-1}+\left(p_{i}-p_{i-1}\right) u, q_{j-1}+\left(q_{j}-q_{j-1}\right) v\right\},
\end{aligned}
$$

where $\left(p_{i}\right)$ and $\left(q_{j}\right)$ are the partitions of $I$ determined by $T$. The following theorem gives two descriptions of the support of $C_{T}$.

Theorem 4. If $T$ is a transformation matrix, which is not a row or column matrix, then the support $\sigma$ of $C_{T}$ satisfies both

(a) $\sigma$ is the invariant set of $\left\{I^{2},\left(f_{i j}\right)^{+}\right\}$, and

(b) $\sigma=\bigcap_{m=1}^{\infty} \sigma_{m}$,

where $\sigma_{m}$ is the support of $T^{m}(\Pi)$.

The plus-sign superscript denotes that the collection is over all $(i, j)$ for which $t_{i j}>0$. Similar notation will be used in other settings to denote the same restriction on $(i, j)$.

Proof. Let $r$ be the maximum of the sidelengths of the $R_{i j}$. Note that $r<1$ and that $\left|f_{i j}(u)-f_{i j}(v)\right| \leq$ $r|u-v|$ for each $u, v \in I^{2}$ and each $(i, j)$. Hence $\left\{I^{2},\left(f_{i j}\right)^{+},\left(t_{i j}\right)^{+}\right\}$is a hyperbolic IFS with probabilities. For any copula $C$ we have $V_{T(C)}(R)=$ $t_{i j} V_{C}\left\{f_{i j}^{-1}(R)\right\}$ for every rectangle $R \subseteq R_{i j}$, and hence $V_{T(C)}(R)=\sum_{i, j}^{+} t_{i j} V_{C}\left\{f_{i j}^{-1}(R)\right\}$ for every rectangle $R \subseteq I^{2}$. Therefore

$\mu_{T(C)}=\sum_{i, j}^{+} t_{i j}\left(\mu_{C} \circ f_{i j}^{-1}\right)=M\left(\mu_{C}\right)$,

where $M$ is the Markov operator of $\left\{I^{2},\left(f_{i j}\right)^{+},\left(t_{i j}\right)^{+}\right\}$. Now $M\left(\mu_{C_{T}}\right)=\mu_{T\left(C_{T}\right)}=\mu_{C_{T}}$, so $\mu_{C_{T}}$ is the invariant measure of $\left\{I^{2},\left(f_{i j}\right)^{+},\left(t_{i j}\right)^{+}\right\}$and (a) follows from 
Proposition 2. It now follows that $\sigma$ is the fixed point of the function $F(K)=\bigcup_{i, j}^{+} f_{i j}(K)$, which is a contraction mapping on the complete metric space of nonempty compact subsets of $I^{2}$ (see Edgar, 1990, proof of Theorem 4.1.3 on p. 107). The ContractionMapping Theorem insures that $\sigma$ is also the limit of the iterative sequence $\left\{F^{m}\left(I^{2}\right)\right\}$, which is the nested sequence $\left(\sigma_{m}\right)$. This establishes (b).

Corollary 1. If $T$ is a transformation matrix with all entries nonzero, then the support of $C_{T}$ is $I^{2}$.

Proof. If $T$ is a row or column matrix, then $C_{T}=$ $\Pi$ from Example 4 . The other cases follow from the preceding theorem as $\sigma_{m}=I^{2}$ for all $m$.

Cuculescu and Theodorescu (2001) use a similar technique to construct copulas, including one whose support is the Sierpinski carpet. The $2 \times 2$ case of the preceding corollary appears in Example 3.3 of the same paper.

Formulas for computing the Hausdorff dimension of the types of sets we have encountered require that the IFSs be similarities. Toward that end, we let $T$ be a transformation matrix that satisfies the following condition:

for each nonzero entry of $T$, the row and column

sumsthrough that entry are equal.

The preceding condition implies that each $R_{i j}$ is a square whenever $t_{i j} \neq 0$, as the relevant column and row sums are $p_{i}-p_{i-1}$ and $q_{j}-q_{j-1}$, respectively. It also implies that $T$ is a square matrix. To see this, suppose that $s$ is a row or column sum and consider the submatrix $S$ of $T$ consisting of the intersections of the rows and columns with sum $s$. Note that all entries in these rows and columns not belonging to $S$ are zero. If $S$ is $m \times n$, then summing its entries by rows and by columns gives $m s=n s$, so $m=n$. It easily follows that $T$ is square.

Definition 3. An invariant copula $C_{T}$ is self-similar if $T$ satisfies (3).

The Borel measures corresponding to self-similar copulas are special cases of self-similar measures (see Edgar, 1998, p. 133). Note that $\Pi, M$ and $W$ are selfsimilar.
For a transformation matrix $T$ satisfying (3), let $r_{i j}$ denote the sidelength of $R_{i j}$ whenever $t_{i j} \neq 0$, and note that the hyperbolic IFS $\left\{I^{2},\left(f_{i j}\right)^{+}\right\}$is a system of similarities with similarity ratios $\left(r_{i j}\right)^{+}$. Since this IFS satisfies Moran's open set condition with open set $U$ equal to the interior of $I^{2}$, the following theorem is a consequence of Theorem 4 and Proposition 1.

Theorem 5. The support of a self-similar copula $C_{T}$ has Hausdorff dimension s given by

$\sum_{i, j}^{+} r_{i j}^{s}=1$

In the following theorem we give sufficient conditions for the support of a self-similar copula to be a fractal.

Theorem 6. Let $C_{T}$ be a self-similar copula for which:

(a) Thas at least one zero entry; and

(b) there is at least one row or column of $T$ with two nonzero entries.

Then the support $\sigma$ of $C_{T}$ is a fractal whose Hausdorff dimension is between 1 and 2 .

Proof. The function $f(s)=\sum_{i, j}^{+} r_{i j}^{s}$ is continuous and strictly decreasing on $[0, \infty)$, as the same holds for each of its terms. Note that $f(2)$ is the sum of the areas of the squares in the support $\sigma_{1}$ of $T(\Pi)$ and that $f(1)$ is the sum of the sidelengths of the squares in $\sigma_{1}$. Hence $f(2)<1$ and $f(1)>1$ by (a) and (b), respectively. It follows from Theorem 5 and the Intermediate-Value Theorem that the Hausdorff dimension of $\sigma$ is in the interval $(1,2)$. Since the topological dimension is an integer which is less than or equal to the Hausdorff dimension (see Edgar, 1990, p. 155), we see that the topological dimension of $\sigma$ is less than or equal to 1 . Hence $\sigma$ is a fractal.

We now consider the family of transformation matrices

$T_{r}=\left[\begin{array}{ccc}r / 2 & 0 & r / 2 \\ 0 & 1-2 r & 0 \\ r / 2 & 0 & r / 2\end{array}\right]$ 
with $r \in(0,1 / 2) . T_{0.2}$ appears in Example 3. It follows from Theorems 5 and 6 that the support of $C_{T_{r}}$ is a fractal whose Hausdorff dimension is the unique solution $s \in(1,2)$ to the equation

$4 r^{s}+(1-2 r)^{s}=1$.

Proposition 5. The function s(r) defined implicitly by (4) is a strictly increasing $C^{\infty}$ bijection from $(0,1 / 2)$ to $(1,2)$.

Proof. For each $s \in(1,2)$ define $g_{s}$ on $(0,1 / 2)$ by $g_{s}(r)=4 r^{s}+(1-2 r)^{s}$. Then $g_{s}^{\prime}(r)=2 s\left\{2 r^{s-1}-\right.$ $\left.(1-2 r)^{s-1}\right\}$, so $g_{s}^{\prime}<0$ on $\left(0, r_{s}\right)$ and $g_{s}^{\prime}>0$ on $\left(r_{s}, 1 / 2\right)$, where $r_{s}=1 /\left\{2+2^{1 /(s-1)}\right\} \in(0,1 / 2)$. Since $g_{s}(0+)=1$ and $g_{s}(1 / 2-)=4 / 2^{s}>1$, there is a unique solution $r \in(0,1 / 2)$ to $g_{s}(r)=1$, i.e., to (4). Hence $s(r)$ is a bijection from $(0,1 / 2)$ to $(1,2)$. To see that $s(r)$ is strictly increasing and $C^{\infty}$ on $(0,1 / 2)$, consider the function $F(r, s)=4 r^{s}+(1-$ $2 r)^{s}$, which is $C^{\infty}$ on $(0,1 / 2) \times(1,2)$. For each $(r, s) \in$ $(0,1 / 2) \times(1,2)$ we have $(\partial F / \partial s)(r, s)=4 r^{s} \ln r+$ $(1-2 r)^{s} \ln (1-2 r)<0$, so $s(r)$ is $C^{\infty}$ on $(0,1 / 2)$ by the Implicit-Function Theorem. Implicit differentiation in (4) yields

$s^{\prime}(r)=\frac{-g_{s}^{\prime}(r)}{\frac{\partial F}{\partial s}(r, s)}$,

which is positive at points $(r, s) \in(0,1 / 2) \times(1,2)$ satisfying (4).

Theorem 1 follows from the fact that $s(r)$ maps $(0,1 / 2)$ onto $(1,2)$.

\section{A generalization of Theorem 1}

Definition 4. A function $f: D \rightarrow \mathbb{R}^{k}$ with $D \subseteq \mathbb{R}^{k}$ is bi-Lipschitz on $D$ if there exist $c_{1}, c_{2}>0$ for which $c_{1}|x-y| \leq|f(x)-f(y)| \leq c_{2}|x-y|$ for all $x, y \in D$.

Note that bi-Lipschitz functions are continuous and one-to-one. Also note that if $f: D \rightarrow \mathbb{R}$ satisfies $0<c_{1} \leq\left|f^{\prime}\right| \leq c_{2}$ on $D$, then $f$ is bi-Lipschitz on $D$ by the Mean-Value Theorem. In addition, note that if $f$ is bi-Lipschitz on $D$, then the inverse of $f$ is bi-Lipschitz on $f(D)$.
Let $F, G: \overline{\mathbb{R}}=[-\infty, \infty] \rightarrow I$ be distribution functions such that for some $a, b, c, d \in \mathbb{R}$

$$
\left\{\begin{array}{l}
F(a)=0, F(b)=1 \text { and } F \text { is bi-Lipschitz on }[a, b], \\
G(c)=0, G(d)=1 \text { and } G \text { is bi-Lipschitz on }[c, d] .
\end{array}\right.
$$

The following lemma is a straightforward consequence of the equivalence of the standard and taxi-cab metrics in the plane.

Lemma 1. If $F$ and $G$ satisfy (5), then the function $F \times G$ defined by $(F \times G)(x, y)=\{F(x), G(y)\}$ is biLipschitz on $[a, b] \times[c, d]$.

Recall from Sklar's Theorem (see Sklar, 1959) that if $H$ is a bivariate distribution function with continuous margins $F$ and $G$, then there is a unique copula $C$ for which $H=C \circ(F \times G)$. Hence

$$
\begin{aligned}
& V_{H}\left(\left[x_{1}, x_{2}\right] \times\left[y_{1}, y_{2}\right]\right) \\
& =V_{C}\left(\left[F\left(x_{1}\right), F\left(x_{2}\right)\right] \times\left[G\left(y_{1}\right), G\left(y_{2}\right)\right]\right) \\
& \quad \text { whenever } x_{1}<x_{2}, y_{1}<y_{2} .
\end{aligned}
$$

Lemma 2. Let $H$ be a bivariate distribution function whose margins $F$ and $G$ satisfy (5). If $S$ is the support of $H$ and $T$ is the support of the copula $C$ associated with $H$, then $(F \times G)(S)=T$.

Proof. Note that $S \subseteq[a, b] \times[c, d]$. Let $S^{c}=$ $([a, b] \times[c, d]) \backslash S, T^{c}=I^{2} \backslash T$ and, for this proof, let $F \times G$ denote the restriction of itself to $[a, b] \times[c, d]$. Recall that $F$ and $G$ are continuous and strictly increasing on $[a, b]$ and $[c, d]$, respectively. If $(x, y)$ is in the interior of a closed rectangle $Q$ in $[a, b] \times[c, d]$ with $V_{H}(Q)=0$, then $(F \times G)(x, y)$ is in the interior of the closed rectangle $(F \times G)(Q)$ and $V_{C}\{(F \times$ $G)(Q)\}=0$ by (6). Since $S$ and $T$ are the closures of their intersections with the interiors of $[a, b] \times[c, d]$ and $I^{2}$, respectively, we see that $(F \times G)\left(S^{c}\right) \subseteq T^{c}$. Since the inverses of the restrictions of $F$ and $G$ to $[a, b]$ and $[c, d]$, respectively, are continuous and strictly increasing on $I$, a similar argument shows that $(F \times G)^{-1}\left(T^{c}\right) \subseteq S^{c}$. The result follows from the fact that $F \times G$ is a bijection from $[a, b] \times$ $[c, d]$ to $I^{2}$. 
We have omitted a long and technical proof that Lemma 2 holds for all continuous margins $F$ and $G$.

Theorem 7. Let $F$ and $G$ be distribution functions which satisfy $(5)$. For each $s \in(1,2)$ there exists a bivariate distribution function $H$ with margins $F$ and $G$, whose support is a fractal of Hausdorff dimension $s$.

Proof. Fix $s \in(1,2)$ and choose, by Theorem 1, a copula $C$ whose support $T$ is a fractal of Hausdorff dimension $s$. Let $H=C \circ(F \times G)$. Then $H$ is a bivariate distribution function with margins $F$ and $G$ by Sklar's Theorem and the support of $H$ is $S=$ $(F \times G)^{-1}(T)$ by Lemma 2 . The function $(F \times G)^{-1}$ is bi-Lipschitz on $I^{2}$ as $F \times G$ is bi-Lipschitz on $[a, b] \times[c, d]$ by Lemma 1 . Hence (see Falconer, 1990 , p. 30) the Hausdorff dimension of $S$ is also $s$. The topological dimension of $S$, being an integer, clearly is less than the Hausdorff dimension of $S$, so $S$ is a fractal.

\section{Acknowledgements}

This work was partially supported by the Ministerio de Ciencia y Tecnología (Spain) and FEDER, under research project BFM2003-06522, and also by the Con- sejería de Educacin y Ciencia of the Junta de Andalucía (Spain).

\section{References}

Barnsley, M.F., 1989. Lecture notes on iterated function systems. In: Devaney, R.L., Keen, L. (Eds.), Chaos and Fractals: The Mathematics Behind the Computer Graphics, Proceedings of Symposia on Applied Mathematics, vol. 39, American Mathematical Society, Providence, pp. 127-144.

Cuculescu, I., Theodorescu, R., 2001. Copulas: diagonals, tracks. Revue roumaine de mathématiques pures et appliquées 46, 731742 .

Edgar, G.A., 1990. Measure, Topology, and Fractal Geometry. Springer, New York.

Edgar, G.A., 1998. Integral, Probability, and Fractal Measures. Springer, New York.

Falconer, K., 1990. Fractal Geometry: Mathematical Foundations and Applications. Wiley, New York.

Li, X., Mikusiński, P., Sherwood, H., Taylor, M.D., 1997. On approximations of copulas. In: Beneš, V., Štěpán, J. (Eds.), Distributions with Given Marginals and Moment Problems. Kluwer, Dordrecht, pp. 107-116.

Mikusiński, P., Sherwood, H., Taylor, M.D., 1992. Shuffles of min. Stochastica 13, 61-74.

Nelsen, R.B., 1999. An Introduction to Copulas. Lecture Notes in Statistics, vol. 139. Springer, New York.

Sklar, A., 1959. Fonctions de répartition à $n$ dimensions et leurs marges. Publications de l'Institut de Statistique de l'Université de Paris 8, 229-231. 